\title{
Silkworm disease diagnosis through molecular approach and their management
}

\author{
B. BEBITHA*, P. MOHANRAJ ${ }^{1}$, S. MANIMEGALAI ${ }^{2}$ AND C.A. MAHALINGAM ${ }^{3}$
}

State Department of Horticulture, Kanchipuram (T.N.) INDIA

${ }^{1}$ Directorate of Extension Education, Tamil Nadu Agricultural University, COIMBATORE (T.N.) INDIA

${ }^{2}$ Department of Sericulture, Tamil Nadu Agricultural University, COIMBATORE (T.N.) INDIA

${ }^{3}$ Department of Entomology, Tamil Nadu Agricultural University, COIMBATORE (T.N.) INDIA

\section{ARITCLE INFO}

Received : 06.02 .2016

Accepted : 25.03.2016

KEY WORDS :

Silkworm disease, Relative humidity,

Photoperiod

*Corresponding author:
How to view point the article : Bebitha, B., Mohanraj, P., Manimegalai, S. and Mahalingam, C.A. (2016). Silkworm disease diagnosis through molecular approach and their management. Internat. J. Plant Protec., 9(1) : 343-352. 